\title{
Accuracy of the Small Field Dosimetry Using the Acuros XB Dose Calculation Algorithm within and beyond Heterogeneous Media for 6 MV Photon Beams*
}

\author{
Sotirios Stathakis, Carlos Esquivel, Luis Vazquez Quino, Pamela Myers, Oscar Calvo, \\ Panayiotis Mavroidis, Alonso N. Gutiérrez, Niko Papanikolaou \\ Departments of Radiology and Radiation Oncology, Cancer Therapy and Research Center, \\ University of Texas Health Science Center, San Antonio, USA \\ Email: Stathakis@uthscsa.edu
}

Received August 2, 2012; revised September 7, 2012; accepted September 17, 2012

\begin{abstract}
Purpose: The dosimetric accuracy of the recently released Acuros XB advanced dose calculation algorithm (Varian Medical Systems, Palo Alto, CA) is investigated for single radiation fields incident on homogeneous and heterogeneous geometries, as well as for two arc (VMAT) cases and compared against the analytical anisotropic algorithm (AAA), the collapsed cone convolution superposition algorithm (CCCS) and Monte Carlo (MC) calculations for the same geometries. Methods and Materials: Small open fields ranging from $1 \times 1 \mathrm{~cm}^{2}$ to $5 \times 5 \mathrm{~cm}^{2}$ were used for part of this study. The fields were incident on phantoms containing lung, air, and bone inhomogeneities. The dosimetric accuracy of Acuros XB, AAA and CCCS in the presence of the inhomogeneities was compared against BEAMnrc/DOSXYZnrc calculations that were considered as the benchmark. Furthermore, two clinical cases of arc deliveries were used to test the accuracy of the dose calculation algorithms against MC. Results: Open field tests in a homogeneous phantom showed good agreement between all dose calculation algorithms and MC. The dose agreement was $+/-1.5 \%$ for all field sizes and energies. Dose calculation in heterogenous phantoms showed that the agreement between Acuros XB and CCCS was within $2 \%$ in the case of lung and bone. AAA calculations showed deviation of approximately $5 \%$. In the case of the air heterogeneity, the differences were larger for all calculations algorithms. The calculation in the patient CT for a lung and bone (paraspinal targets) showed that all dose calculation algorithms predicted the dose in the middle of the target accurately; however, small differences $(2 \%-5 \%)$ were observed at the low dose region. Overall, when compared to MC, the Acuros XB and CCCS had better agreement than AAA. Conclusions: The Acuros XB calculation algorithm in the newest version of the Eclipse treatment planning system is an improvement over the existing AAA algorithm. The results are comparable to CCCS and MC calculations especially for both stylized and clinical cases. Dose discrepancies were observed for extreme cases in the presence of air inhomogeneities.
\end{abstract}

Keywords: Photon Dose Calculation; Inhomogeneities; Acuros XB

\section{Introduction}

Recent technological advances in the dose delivery, positioning, and immobilization of the patient have led to more complex treatment plans such as intensity modulated radiation therapy (IMRT) and stereotactic body radiotherapy (SBRT). IMRT was introduced in the 1990s and produces highly conformal distributions with steep dose gradients and improved sparing of normal tissue and critical organs. This is achieved through intensity modulated beams and the delivery of the dose through a series of subfields (segments) within each field. Stereotactic body radiotherapy refers to the precise irradiation

\footnotetext{
${ }^{*}$ Conflict of interest statement: There are no conflicts of interest with regards to this manuscript.
}

of an image-defined extracranial lesion using a small number (one to five) of high-dose fractions. SBRT shares characteristics of both radiosurgery and conventionally fractionated radiotherapy (CFR). The high doses per fraction are meant to have an ablative tumor effect, whereas the use of modest fractionation implies the clinical need to allow for some normal tissue recovery. Usually, the target volumes for SBRT are relatively small compared to CFR, hence, radiation field sizes are typically smaller than the ones used for traditional radiation therapy. The lesions treated can be in proximity to vital sensitive structures and in heterogenous media. Therefore, the accuracy of treatment planning and dose delivery is very important. The aim of SBRT is to deliver dose to the target with a positional accuracy of $<1 \mathrm{~mm}$ and ac- 
curacy in dose of $<5 \%$ with steep dose falloff outside the target volume. The treatment plan can be $3 \mathrm{D}$ based with unmodulated noncoplanar beams or IMRT. In either case, the effect of the inhomogeneity in the dose calculation can be significant. Given the high dose per fraction for SBRT treatments, the need for an accurate dose computation is further elevated. Although for SBRT in the liver a pencil beam-type calculation may be adequate, the same is not true for SBRT in the lung or for paraspinal lesions.

Under such conditions of small field geometries, the electronic equilibrium can be lost, making it challenging for the dose-calculation algorithm to accurately predict the dose, especially in the presence of tissue heterogeneities. In narrow photon beams the absorbed dose changes rapidly with beam size and depth in a phantom $[1,2]$. This is attributed to the absence of both lateral and longitudinal electronic equilibrium when the dimensions of the radiation field are smaller than the maximum range of secondary electrons. These relatively large areas of electronic disequilibrium in such narrow photon beams fields make accurate dosimetry more difficult than with conventional radiotherapy beams. This disequilibrium effect can be exacerbated in areas of tissue heterogeneity. Brain tissue heterogeneity is not considered in stereotactic radiosurgery. However, as stereotactic radiotherapy is applied to extracranial areas such as the head and neck and lung, dose calculations need to account for dose perturbations in and beyond air cavities, lung tissue, and bone.

When calculating the dose in a low-density medium such as lung using narrow beams, tissue density variations can introduce significant perturbations that are energy and density dependent and affect the accuracy of the dose calculation. This problem is more pronounced when the treatment planning system (TPS) uses simple, onedimensional density scaling [3-6]. The level of accuracy improves with the use of more sophisticated treatment planning algorithms [7-14] where multisource modeling is included, allowing a more accurate dose prediction for small fields and non-equilibrium conditions $[1,2]$. It has been shown that the accuracy of small field dosimetry is greatly improved when Monte Carlo calculations are used, especially for beam with apertures less than $3 \times 3$ $\mathrm{cm}^{2}$ in homogeneous media [15-18]. IMRT planning utilizes iterative optimization techniques, forcing the dose to be re-calculated after each iteration.

An alternate approach to the Monte Carlo method was recently developed that is based on the deterministic solution of the linear Boltzmann transport equation (LBTE). A benefit of the deterministic radiation transport solutions of the LTBE compared to Monte Carlo simulations, is the absence of statistical noise in the calculated dose. Such algorithm for external photon beam treatment planning has been developed and has been implemented in the Varian Eclipse external beam treatment planning system (TPS) (Varian Medical Systems, Palo Alto, CA, USA). This new algorithm is called Advanced Dose Calculation algorithm (Acuros XB) and is the subject of this investigation. The first clinical release of Acuros XB for external beam planning became available in Eclipse version 10.0 .

In this study, we are comparing the Acuros XB dose calculation algorithm against existing commercial dose calculation algorithms such as the Analytical Anisotropic Algorithm (AAA), Collapsed Cone Convolution Superposition (CCCS), and Monte Carlo dose calculations. To this date, only one paper has presented results between the four dose calculation methods for fields $2.5 \times 2.5 \mathrm{~cm}^{2}$ and larger [19]. Other studies have been published where comparison of the Acuros XB to AAA or Monte Carlo is available but no comparison with CCCS [20-22]. Furthermore, the Acuros XB has been compared and evaluated for its accuracy for small fields but the comparison did not include the CCCS. With this study we are trying to fill the gap in the literature by providing a comparison between all four dose calculation algorithms of small fields in heterogeneous media.

\section{Materials and Methods}

\subsection{Monte Carlo Commissioning}

A Monte Carlo model of the linear accelerator was commissioned to simulate the linear accelerator head with the intent to use it for dose calculations. The EGSn rc\BEAMnrc [23] and EGSnrc\DOSXYZnrc [24] packages were used for the Monte Carlo calculations. For this study, the $6 \mathrm{MV}$ photon beam was simulated for a Varian NovalisTX ${ }^{\mathrm{TM}}$ using various open field sizes $(1 \times 1,2 \times 2,3 \times 3$, $4 \times 4,6 \times 6,8 \times 8$, and $\left.10 \times 10 \mathrm{~cm}^{2}\right)$ of data measured in water at an SSD $=100 \mathrm{~cm}$. The PTW (PTW Freiburg $\mathrm{GmbH}$, Germany) microLion liquid ionization chamber (vol $=0.002 \mathrm{cc}$ ) was used for the measurements. The Monte Carlo model of the Novalis TX was validated against these measurements. Percent depth dose curves PDD) and dose profiles at different depths were calculated and compared with measurements.

Geometry and materials used to build the Monte Carlo model of the linear accelerator were based on machine specifications as provided by the manufacturer. The linac was structured in the following order: a target slab of tungsten and copper, primary collimator made of tungsten, flattening filter, ion chamber, jaws (tungsten), and the Varian MLC (VARMLC) (Figure 1). All materials used in the MC simulation were extracted from the 700 ICRU PEGS4 (Preprocessor for Electron Gamma Shower) cross section data available in BEAMnrc and met the specifications for the linac as provided by the manufac- 


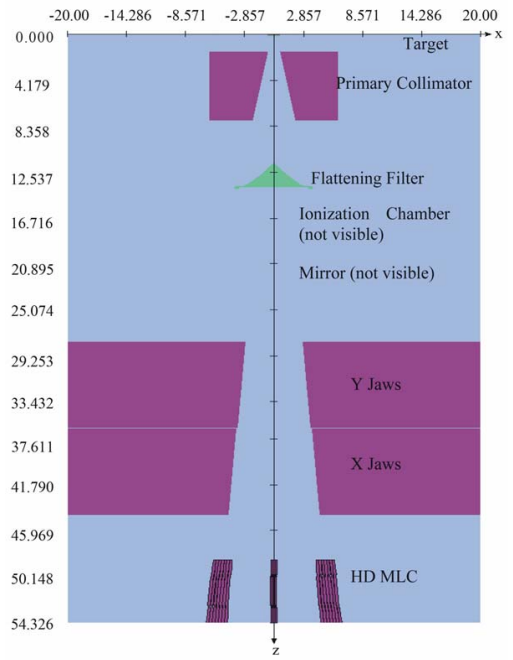

Figure 1. BeamNRC Monte Carlo model for the simulation of the Novalis TX with the HD MLC.

turer. The cutoff energies used in the simulations were ECUT $=700 \mathrm{KeV}$ for electrons and PCUT $=10 \mathrm{KeV}$ for photons. A monoenergetic, $6 \mathrm{MeV}$ source was in the simulation used with full width at half maximum (FWHM) for the $\mathrm{X}$ and $\mathrm{Y}$ directions of $0.125 \mathrm{~cm}$.

Phase space files from BEAMnrc were used as source files in DOSXYZnrc to calculate the dose in a water phantom designed for this purpose with voxel dimensions of $0.3 \times 0.3 \times 0.3 \mathrm{~cm}^{3}$, and phantom dimension of $30 \times 30 \times 30 \mathrm{~cm}^{3}$. DOSXYZnrc simulations in the water phantom were executed with high enough number of histories to give a statistical uncertainty in the dose calculation of the twenty highest doses of less than $1 \%$.

\subsection{Dose Calculations and TPS}

\subsubsection{Phantoms}

For the evaluation of the Acuros XB dose calculation algorithm, inhomogeneities were introduced in a homogeneous water phantom, as shown in Figure 2. The inhomogeneities were placed at $5 \mathrm{~cm}$ depth, and extended to $10 \mathrm{~cm}$ depth while the lateral dimensions were either $15 \times 15 \mathrm{~cm}^{2}$ or $15 \times 7.5 \mathrm{~cm}^{2}$. The inhomogeneity media were composed of lung, air and bone (see Table 1) Furthermore, a patient CT data set was used for the clinical simulations.

\subsubsection{Test Cases}

The phantoms in Figure 2 were used to calculate the dose distributions for field sizes ranging from $1 \times 1$ to $10 \times$ $10 \mathrm{~cm}^{2}$. The PDDs and profiles at the locations marked with dashed lines in Figure 2 were extracted for comparison. The dose calculations were obtained using Monte Carlo, the Acuros XB, the analytical anistropic algori-

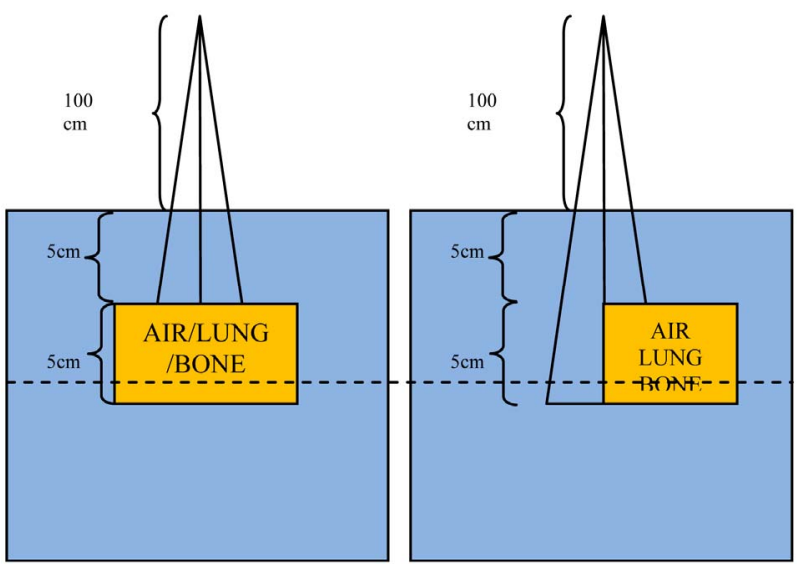

Figure 2. Slab geometry phantom used for simulations and calculations in the TPS.

Table 1. Mass density and electron density of material used inside the slab geometry Monte Carlo simulations and TPS calculations.

\begin{tabular}{cccc}
\hline Media & Mass density & Electron density & CT number \\
\hline Water & 1.0 & 1.0 & 5 \\
Lung & 0.3 & 0.29 & -725 \\
Air & 0.001 & 0.001 & -1000 \\
Bone & 1.5 & 1.472 & 875 \\
\hline
\end{tabular}

thm (AAA) available in Eclipse, and the collapsed cone convolution superposition (CCCS) algorithm avail- able in Oncentra Master Plan treatment planning system (OMP).

The patient CT shown in Figure 3 was used for the dose calculation in two different scenarios: one of lung SBRT and one of spine SBRT. For these cases, arcs of $1 \times$ $1,2 \times 2$ and $3 \times 3 \mathrm{~cm}^{2}$ were calculated with the isocenter at the center of each of the targets in the lung and spine respectively. The scope of this investigation was not to report on the plan quality but to test the dose calculation algorithms in near real planning cases.

All TPS are commissioned for our Novalis TX linear accelerator. The commissioning of all systems was completed according to each TPS manufacturer instructions which included the acquisition of PDDs, profiles and output factors.

\subsubsection{Evaluation}

Profiles and PDDs were compared against the Monte Carlo, Acuros XB, AAA and CCCS calculations for all geometries shown in Figure 2. The profiles were generated at the positions shown in Figures 2 and 3. For the lung and spine SBRT, profiles were obtained through the isocenter for the axial, coronal and sagittal planes. In order to remove bias in the comparison between the dose 

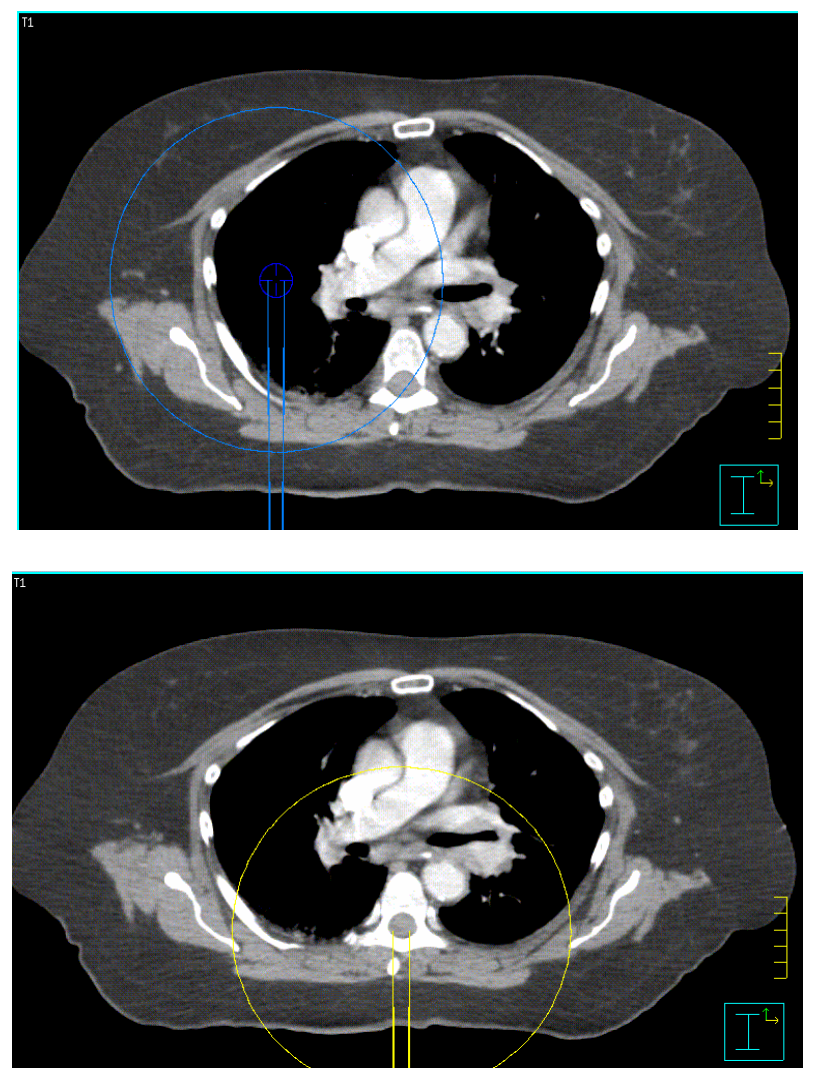

Figure 3. Isocenter locations for the spinal cord and lung SmartArc deliveries.

calculations, the dose grid resolution was set to $0.3 \mathrm{~cm} \times$ $0.3 \mathrm{~cm} \times 0.3 \mathrm{~cm}$ for all calculations.

\section{Results}

\subsection{Monte Carlo Commissioning}

The Monte Carlo calculations for the commissioning of the linac were in good agreement with the measurements in water (Figures 4 and 5). The agreement was within $1 \%$ and $1 \mathrm{~mm}$ for all PDDs and profiles calculated. Based on this finding the Monte Carlo model of the $6 \mathrm{MV}$ photon beam was used as the gold standard for all the dosimetric comparisons against the other algorithm used in this study.

The dose for the $1 \times 1$ to $5 \times 5 \mathrm{~cm}^{2}$ fields using the 6 MV photon beam in the slab geometry shown in Figure 2 were calculated for all four methods. The percent depth dose and a profile at $7.5 \mathrm{~cm}$ depth perpendicular to the central axis are shown in Figures 6 and 7 Dose calculations were obtained for the different density heterogeneities for each dose calculation algorithm and jaw setting. The highest discrepancies between the different methods against the Monte Carlo are observed in the case of the air heterogeneity. AcurosXB calculations were in good agreement with MC for lung and bone inhomogeneities. The differences were in most cases within 3\% or $1 \mathrm{~mm}$ in the high dose gradient areas. Similar results were obtained for the CCCS calculation while AAA showed differences exceeding 5\% for the smallest fields in bone and lung inhomogeneity. In the case of the air inhomogeneity, the difference between AAA and MC was about $50 \%$ for the $1 \times 1 \mathrm{~cm}^{2}$ traversing though a slab of air. For the same geometry, Acuros XB and CCCS showed differences that ranged from $3 \%$ to $15 \%$.

In terms of the dose at the interface between the bone and water and lung and water all calculation algorithms showed small differences against $\mathrm{MC}$ calculations. The differences were less than $2 \%$ for Acuros XB and CCCS and less than 5\% for AAA (Figures 6 and 7).

It is shown in Figure 7 that no calculation algorithm was able to accurately predict the dose when the air heterogeneity was partially inside the field (Figure 2(b)). Our calculations show that the differences against MC increased with increasing field size. The magnitude of the differences between the dose calculation algorithms and Monte Carlo ranged from $2 \%$ to $50 \%$ depending on the dose calculation algorithm and field size, with the AAA performing the poorest while Acuros XB performed the best.

\subsection{Arc Delivery}

For a clinical assessment of the accuracy of the four algorithms, we calculated six plans using full $360^{\circ}$ degree arcs on patient CT image sets. The fields were not defined by MLC but rather used jaws, with field sizes of 11, $2 \times 2$, and $3 \times 3 \mathrm{~cm}^{2}$. The dose calculations for the lung and spine targets were compared against the Monte Carlo. Profiles at the isocenter plane through the middle of the targets are shown in Figures 8(a) and (b). The high dose region was in very good agreement with Monte Carlo for all cases. However, discrepancies were observed in the low dose region where the dose predicted by the TPSs was lower than the one predicted by Monte Carlo calculations. The spinal cord plans showed larger differences than the lung plans in the low dose region. In all the clinical scenarios that we simulated, the differences did not exceed $7 \%$.

Figure 8(b) shows absolute dose profile for the spinal canal target for the $2 \times 2 \mathrm{~cm}^{2}$ arc. Differences at the center of the profile can be seen in this case between the four dose calculations algorithms. The differences can be attributed to the fact that AAA computes the transport and dose deposition using radiological density scaling and reports dose in water while, Acuros XB calculates the dose considering the elemental composition of tissues and reports the dose to the medium.

\section{Discussion}

In this study, Acuros XB version 10.0 was validated against $\mathrm{MC}$ and two other clinical convolution methods 


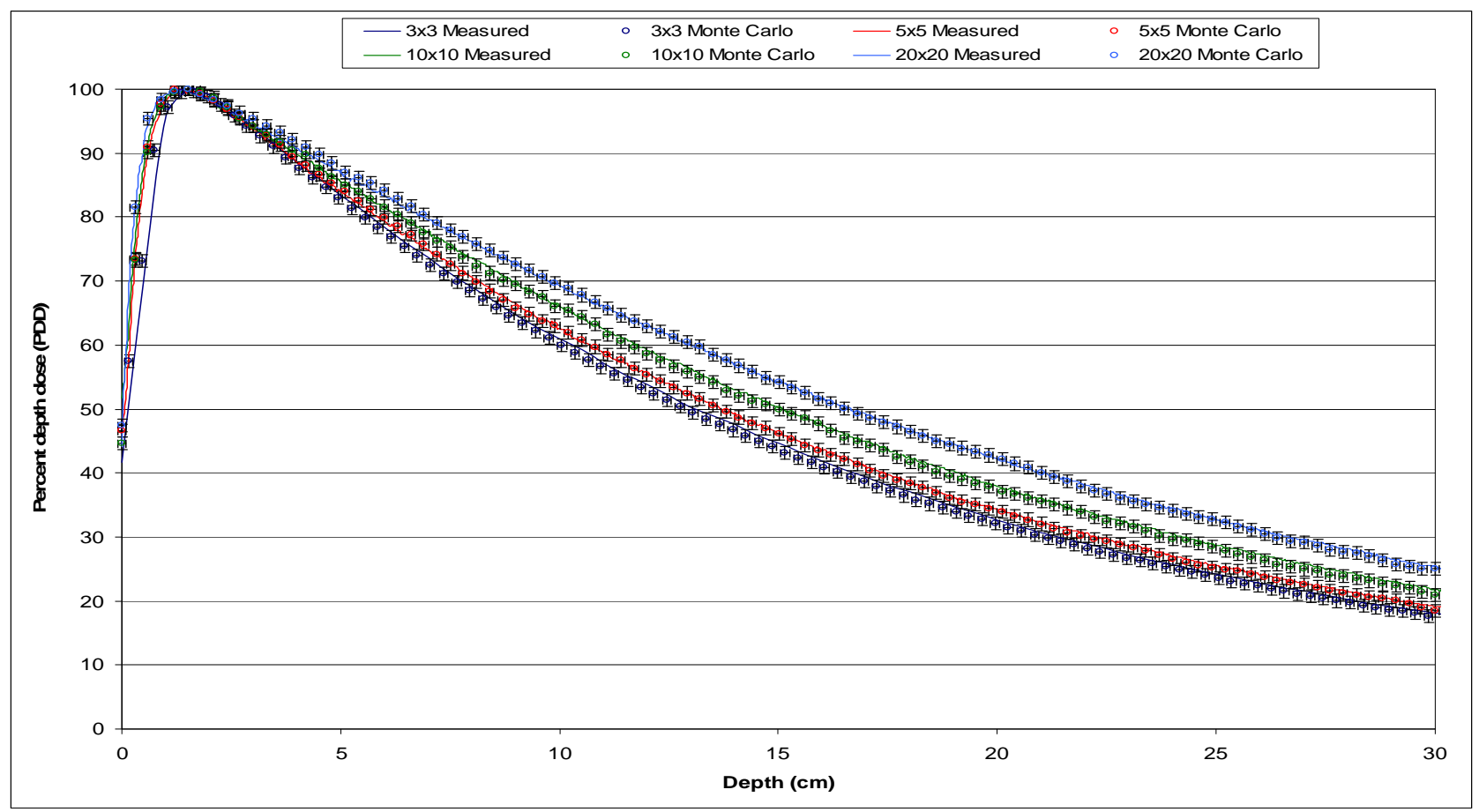

Figure 4. PDDs comparison for $3 \times 3 \mathrm{~cm}^{2}, 5 \times 5 \mathrm{~cm}^{2}, 10 \times 10 \mathrm{~cm}^{2}$, and $20 \times 20 \mathrm{~cm}^{2}$ field sizes defined by jaws. Solid lines represent ion chamber measurements and circles with error bars of $1 \%$ and $1 \mathrm{~mm}$ represent Monte Carlo results.

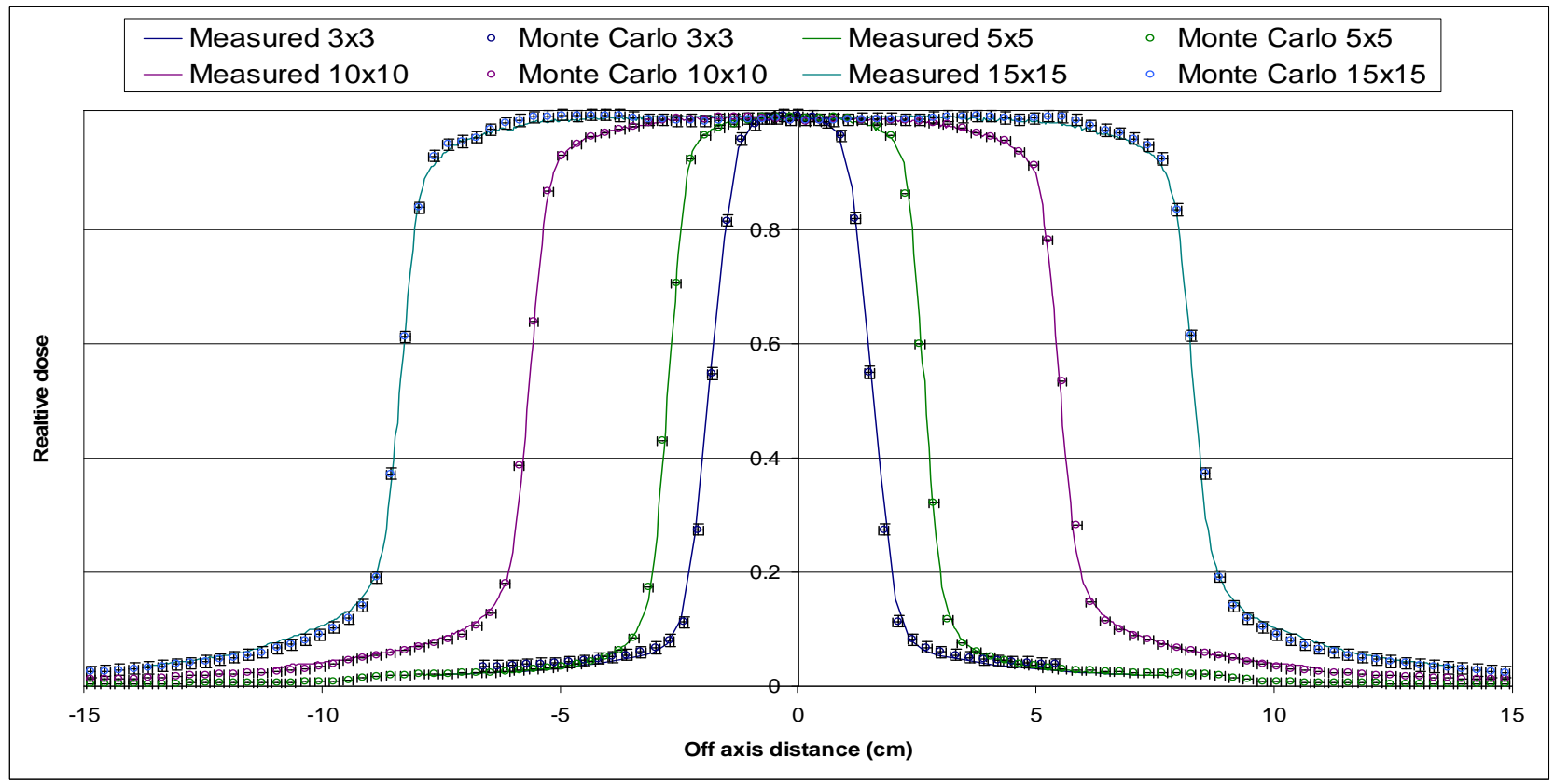

Figure 5. Profile for different field sizes defined by HD-MLC at $10 \mathrm{~cm}$ depth in water phantom. $3 \times 3 \mathrm{~cm}^{2}, 5 \times 5 \mathrm{~cm}^{2}, 10 \times 10 \mathrm{~cm}^{2}$ and $15 \times 15 \mathrm{~cm}^{2}$ fields. Solid lines represent measured dose and circles with error bars (1\% and $\left.1 \mathrm{~mm}\right)$ Monte Carlo simulations.

(AAA and CCCS). Our benchmark was primarily focused on comparisons of depth dose and lateral dose profiles of jaw defined narrow photon beams in both a homogeneous water and heterogeneous slab phantoms. Our results for bone and lung heterogeneities are in agree ment with previously reported data $[19,20]$. Based on our findings: 1) all the dose calculation algorithms are in good agreement with MC (within $2 \%$ for CCCS and AcurosXB and 5\% for AAA); 2) the differences are more pronounced at the interfaces between the inhomogeneities; and 3) AAA shows the larger discrepancies when compared against $\mathrm{MC}$ calculations. 
Large differences were observed in the case of the air heterogeneity. The differences were shown both in the axial profiles and when the inhomogeneity covered half of the field (Figure 7). The differences along the central
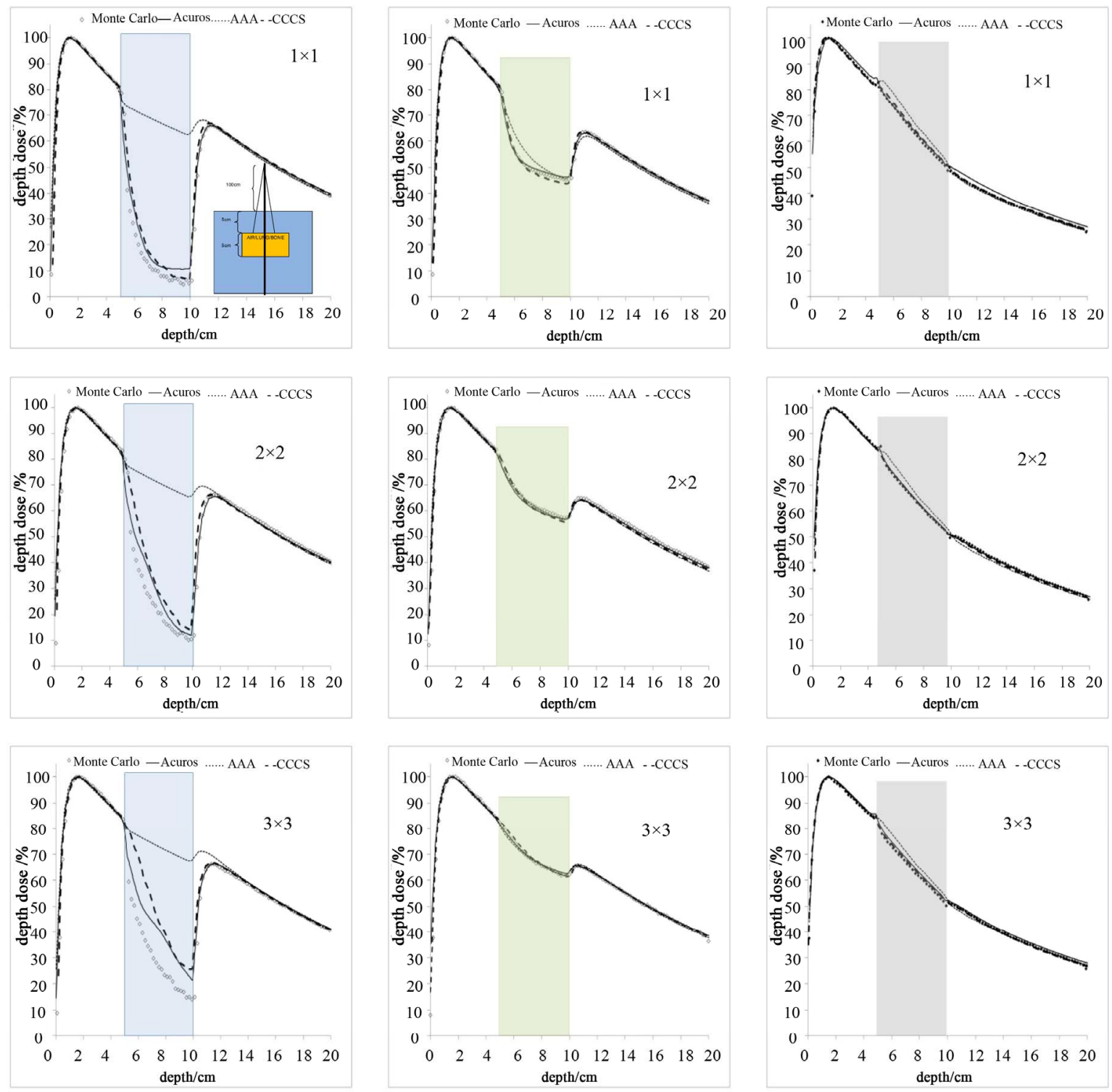

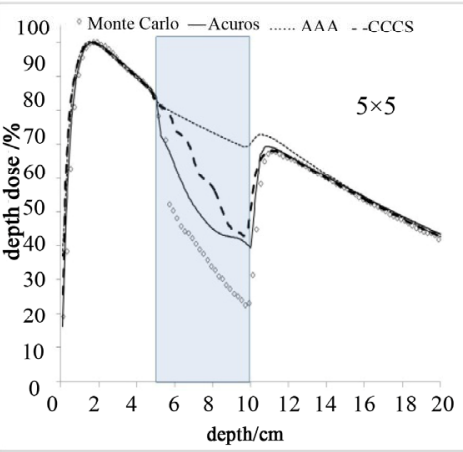

(a)

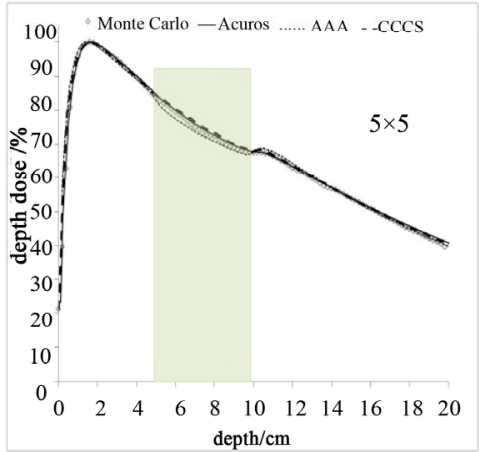

(b)

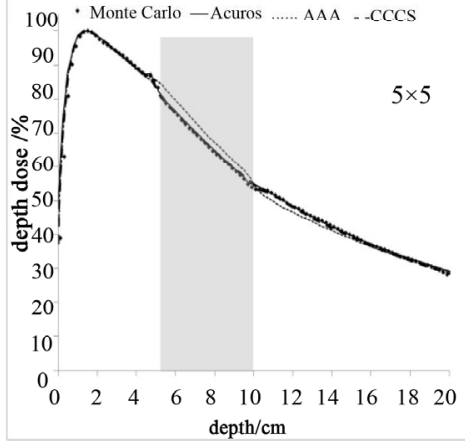

(c)

Figure 6. PDD curves for 3 different inhomogeneities. Column (a): air, Column (b): lung, and Column (c): bone for various small field sizes. 

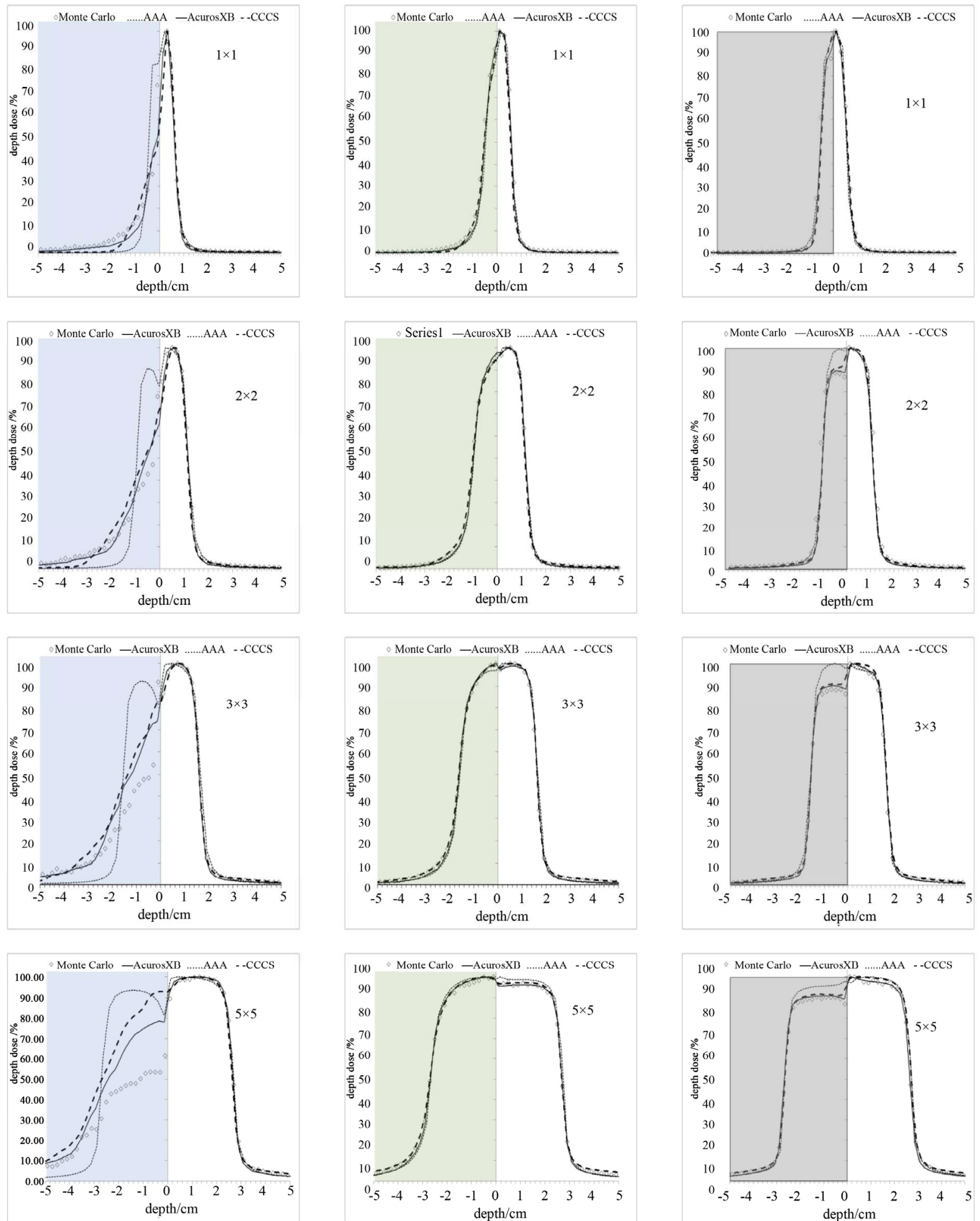

(a)

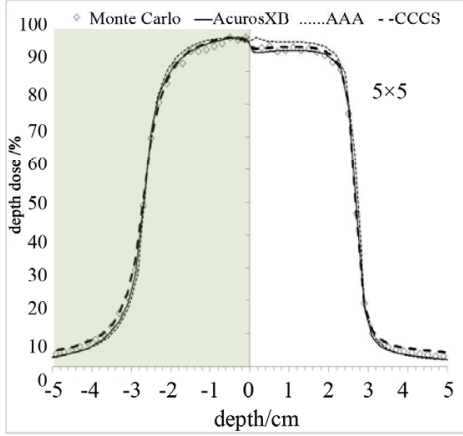

(b)

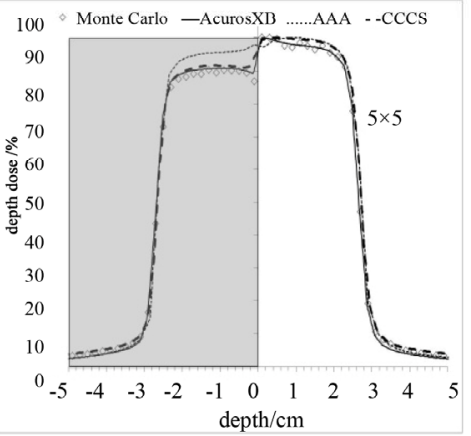

(c)

Figure 7. Profiles for 3 different inhomogeneities. Column (a): air, Column (b): lung, and Column (c): bone for various small field sizes at inside the inhomogeneity.

axis profile were higher: 1) for AAA for all fields tested, 2) for the larger field sizes. These differences range from $5 \%$ to $50 \%$ depending on the dose calculation algorithm, and field size. In this case, the AAA algorithm failed to predict the perturbation of the dose due to the presence of the inhomogeneity resulting in overestimation of dose in 

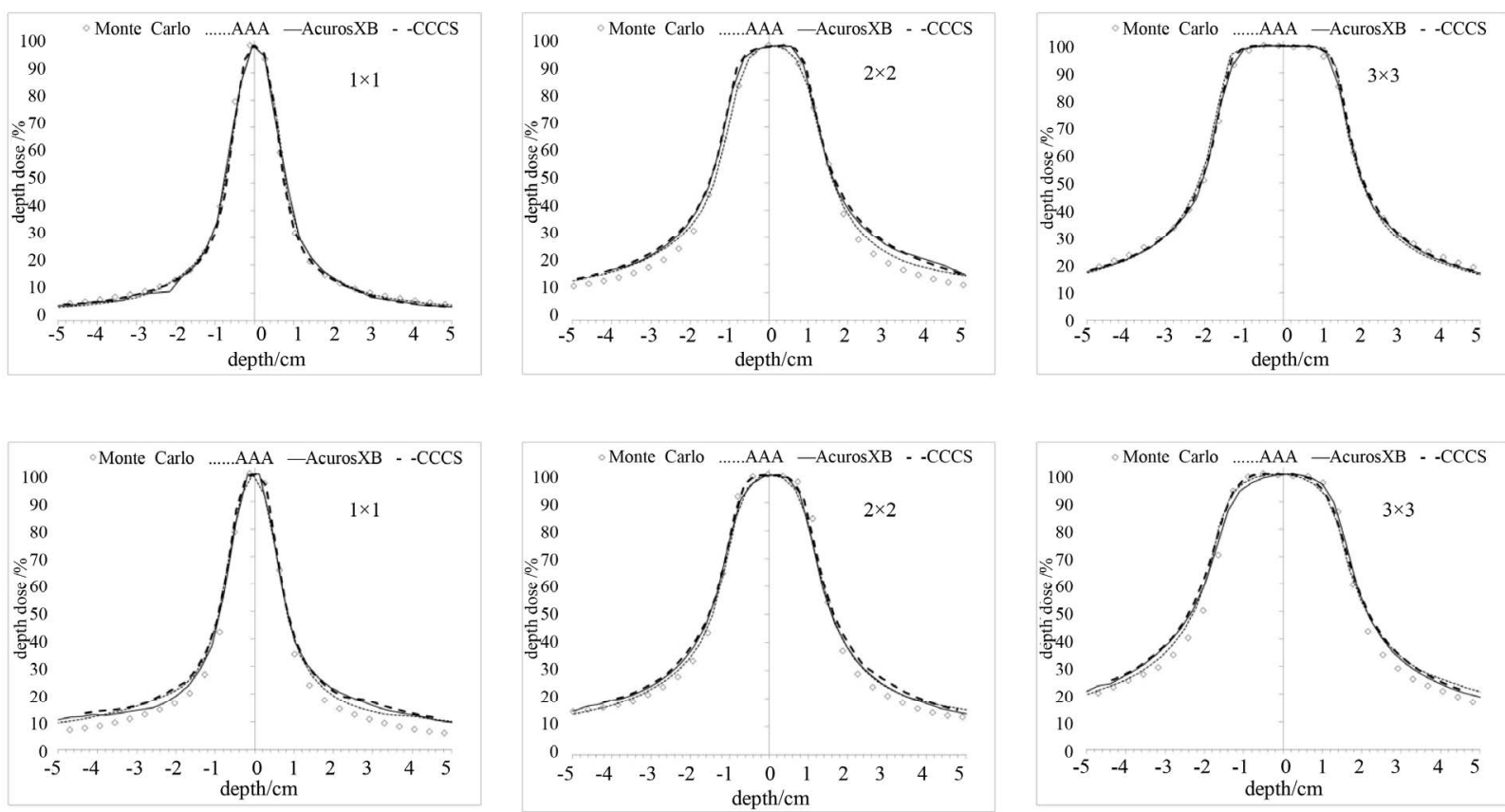

(a)

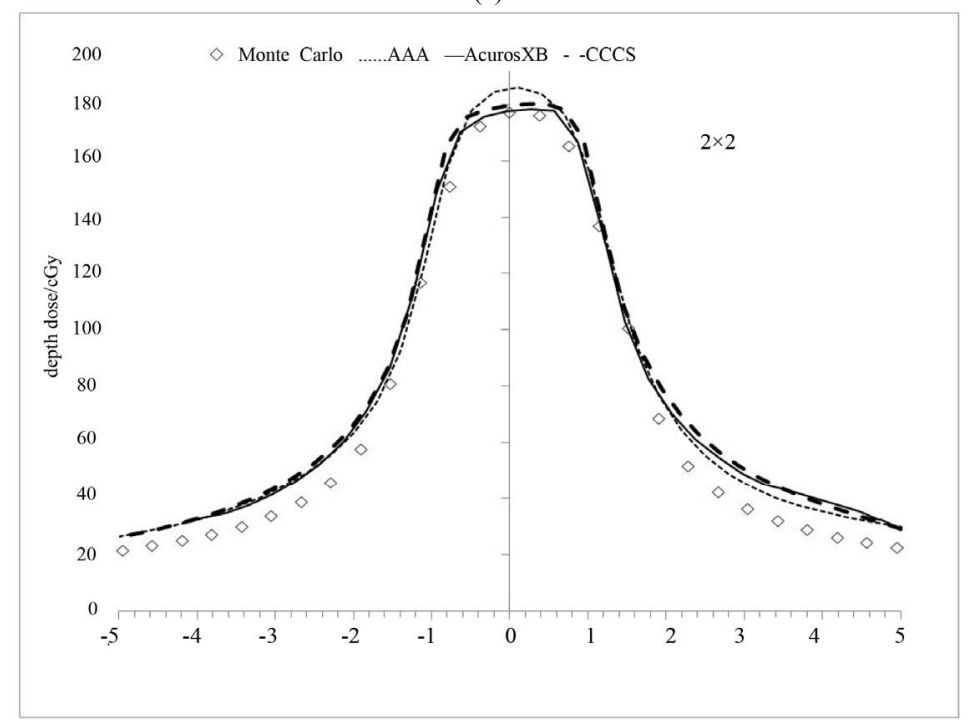

(b)

Figure 8. (a) Dose profiles for a complete arc delivery of a lung target (top) and spinal canal target (bottom) at the plane of the isocenter; (b) Absolute dose profile for the spinal canal target using $2 \times 2 \mathrm{~cm}^{2}$.

that region.

We found that for the half field inhomogeneity (Figure 2(b)) the discrepancies at the interface and inside the inhomogeneity: 1) AAA calculations had the worst agreement with MC and 2) the air inhomogeneity showed the worst agreement for all calculation methods.

For the CT based arc deliveries for the lung and spinal cord targets, all methods were in good agreement with the $\mathrm{MC}$ for the in-field analysis. Discrepancies were observed in the low dose regions outside the field. These discrepancies are similar for all dose calculation algorithms and no one algorithm clearly outperformed the other ones. These results are in agreement with the results from the slab geometry experiments with bone and lung inhomogeneities.

The results presented here do include statistical uncertainties observed during our Monte Carlo calculations but no such uncertainties are easily available for dose calculation algorithms. Although is easy to report uncertainties for Monte Carlo dose calculations, it is fairly difficult to report uncertainties for commercial dose calculations. However, one can test such dose calculation algorithms against measurements and/or Monte Carlo calculations. Gray et al. reported that they observed errors 
greater than $2.5 \%$ when using AAA to calculate the dose beyond a large air gap compared to measurements [25]. Bush et al. observed differences between AAA and MC methods within the range of $2.5 \%$ and $9.6 \%$ [26]. Carassco et al. have reported uncertainties of about $1.3 \%$ for calculations in lung phantoms with CCCS compared to Monte Carlo and measurements [27]. Dvorak et al. observed deviations of about $2 \%$ when IMRT plans were recalculated with the CCCS algorithm, especially for the high nominal energy and compared to measurements [28].

\section{Conclusion}

The Acuros XB advanced dose calculation algorithm in the Eclipse TPS produced results that are comparable to the $\mathrm{MC}$ method. In particular, we found that Acuros XB improves the dose prediction accuracy over both AAA and CCCS in the presence of both bone and lung heterogeneities. Acuros XB dose predictions near an inter face are in closer agreement to MC than both AAA and CCCS. Discrepancies were observed in water/air interfaces. In this case, the Acuros XB had a better agreement with MC while AAA had the worst agreement. All algorithms performed poorly when predicting the dose at the interfaces. For the clinical simulation cases of arc deliveries for a lung and a spinal cord target, all methods produced similar results.

\section{REFERENCES}

[1] T.C. Zhu and B. E. Bjarngard, "The Fraction of Photons Undergoing Head Scatter in X-Ray Beams," Physics in Medicine and Biology, Vol. 40, No. 6, 1995, pp. 11271134. doi:10.1088/0031-9155/40/6/011

[2] T. C. Zhu and B. E. Bjarngard, "The Head-Scatter Factor for Small Field Sizes," Medical Physics, Vol. 21, No. 1, 1994, pp. 65-68. doi:10.1118/1.597256

[3] T. R. Mackie, J. W. Scrimger, J. J. Battista, "A Convolution Method of Calculating Dose for 15-MV X-Rays," Medical Physics, Vol. 12, No. 2, 1985, pp. 188-196. doi:10.1118/1.595774

[4] H. A. Al-Hallaq, C. S. Reft and J. C. Roeske, "The Dosimetric Effects of Tissue Heterogeneities in IntensityModulated Radiation Therapy (IMRT) of the Head and Neck," Physics in Medicine and Biology, Vol. 51, No. 5, 2006, pp. 1145-1156. doi:10.1088/0031-9155/51/5/007

[5] I. J. Chetty, P. M. Charland, N. Tyagi, et al., "Photon Beam Relative Dose Validation of the DPM Monte Carlo Code in Lung-Equivalent Media," Medical Physics, Vol. 30, No. 4, 2003, pp. 563-573. doi:10.1118/1.1555671

[6] H. Saitoh, T. Fujisaki, R. Sakai, et al., "Dose Distribution of Narrow Beam Irradiation for Small Lung Tumor," International Journal of Radiation Oncology, Biology, Physics, Vol. 53, No. 5, 2002, pp. 1380-1387.

[7] A. L. Boyer and E. C. Mok, "Calculation of Photon Dose Distributions in an Inhomogeneous Medium Using Convolutions," Medical Physics, Vol. 13, No. 4, 1986, pp. 503-

\section{9. doi:10.1118/1.595964}

[8] C. M. Bragg and J. Conway, "Dosimetric Verification of the Anisotropic Analytical Algorithm for Radiotherapy Treatment Planning," Radiotherapy and Oncology: Journal of the European Society for Therapeutic Radiology and Oncology, Vol. 81, No. 3, 2006, pp. 315-323.

[9] J. Craig, M. Oliver, A. Gladwish, et al., "Commissioning a Fast Monte Carlo Dose Calculation Algorithm for Lung Cancer Treatment Planning," Journal of Applied Clinical Medical Physics/American College of Medical Physics, Vol. 9, No. 2, 2008, p. 2702.

[10] I. M. Gagne and S. Zavgorodni, "Evaluation of the Analytical Anisotropic Algorithm in an Extreme Water-Lung Interface Phantom Using Monte Carlo Dose Calculations," Journal of Applied Clinical Medical Physics/American College of Medical Physics, Vol. 8, No. 1, 2007, pp. 3346.

[11] F. Garcia-Vicente, A. Minambres, I. Jerez, et al., "Experimental Validation Tests of Fast Fourier Transform Convolution and Multigrid Superposition Algorithms for Dose Calculation in Low-Density Media," Radiotherapy and Oncology: Journal of the European Society for Therapeutic Radiology and Oncology, Vol. 67, No. 2, 2003, pp. 239-249.

[12] E. Kunieda, H. M. Deloar, N. Kishitani, et al., "Variation of Dose Distribution of Stereotactic Radiotherapy for Small-Volume Lung Tumors under Different Respiratory Conditions," Physica Medica, Vol. 24, No. 4, 2008, pp. 204-211.

[13] C. Scholz, C .Schulze and U. Oelfke, et al., "Development and Clinical Application of a Fast Superposition Algorithm in Radiation Therapy," Radiotherapy and Oncology: Journal of the European Society for Therapeutic Radiology and Oncology, Vol. 69, No. 1, 2003, pp. 79-90.

[14] L. Tillikainen, H. Helminen, T. Torsti, et al., "A 3D Pencil-Beam-Based Superposition Algorithm for Photon Dose Calculation in Heterogeneous Media," Physics in Medicine and Biology, Vol. 53, No. 14, 2008, pp. 3821-3839. doi:10.1088/0031-9155/53/14/008

[15] A. Ahnesjo, "Collimator Scatter in Photon Therapy Beams," Medical Physics, Vol. 22, No. 3, 1995, pp. 267-278. doi: $10.1118 / 1.597450$

[16] G. X. Ding, "Dose Discrepancies between Monte Carlo Calculations and Measurements in the Buildup Region for a High-Energy Photon Beam," Medical Physics, Vol. 29, No. 11, 2002, pp. 2459-2463. doi:10.1118/1.1514237

[17] G. X. Ding, D. M. Duggan and C. W. Coffey, "Commissioning Stereotactic Radiosurgery Beams Using both Experimental and Theoretical Methods," Physics in Medicine and Biology, Vol. 51, No. 10, 2006, pp. 2549-2566. doi:10.1088/0031-9155/51/10/013

[18] P. K. Kijewski, B. E. Bjarngard and P. L. Petti, "Monte Carlo Calculations of Scatter Dose for Small Field Sizes in a 60 Co Beam," Medical Physics, Vol. 13, No. 1, 1986, pp. 74-77. doi:10.1118/1.595925

[19] T. Han, J. K. Mikell, M. Salehpour, et al., "Dosimetric Comparison of Acuros XB Deterministic Radiation Transport Method with Monte Carlo and Model-Based Convolution Methods in Heterogeneous Media," Medical Phys- 
ics, Vol. 38, No. 5, 2011, pp. 2651-2664. doi:10.1118/1.3582690

[20] A. Fogliata, G. Nicolini, A. Clivio, et al., "On the Dosimetric Impact of Inhomogeneity Management in the Acuros XB Algorithm for Breast Treatment," Radiation Oncology, Vol. 6, No. 1, 2011, p. 103. doi:10.1186/1748-717X-6-103

[21] A. Fogliata, G. Nicolini, A. Clivio, et al., "Dosimetric Evaluation of Acuros XB Advanced Dose Calculation Algorithm in Heterogeneous Media," Radiation Oncology, Vol. 6, No. 1, 2011, p. 82. doi:10.1186/1748-717X-6-82

[22] K. Bush, I. M. Gagne, S. Zavgorodni, et al., "Dosimetric Validation of Acuros XB with Monte Carlo Methods for Photon Dose Calculations," Medical Physics, Vol. 38, No. 4, 2011, pp. 2208-2221. doi:10.1118/1.3567146

[23] D. W. Rogers, B. A. Faddegon, G. X. Ding, et al., "BEAM: A Monte Carlo Code to Simulate Radiotherapy Treatment Units," Medical Physics, Vol. 22, No. 5, 1995, pp. 503524. doi:10.1118/1.597552

[24] B. Walters and I. Kawrakow, "Rogers DWO: DOSXYZnrc User Manual,” NRCC Report PIRS-794revB, 2009.
[25] A. Gray, L. D. Oliver and P. N. Johnston, "The Accuracy of the Pencil Beam Convolution and Anisotropic Analytical Algorithms in Predicting the Dose Effects Due to Attenuation from Immobilization Devices and Large Air Gaps," Medical Physics, Vol. 36, No. 7, 2009, pp. 31813191. doi:10.1118/1.3147204

[26] K. Bush, S. Zavgorodni, I. Gagne, et al., "Monte Carlo Evaluation of RapidArc Oropharynx Treatment Planning Strategies for Sparing of Midline Structures," Physics in Medicine and Biology, Vol. 55, No. 16, 2010, pp. 44654479. doi:10.1088/0031-9155/55/16/S03

[27] P. Carrasco, N. Jornet, M. A. Duch, et al., "Comparison of Dose Calculation Algorithms in Slab Phantoms with Cortical Bone Equivalent Heterogeneities," Medical Physics, Vol. 34, No. 8, 2007, pp. 3323-3333. doi:10.1118/1.2750972

[28] P. Dvorak, M. Stock, B. Kroupa, et al., "Analysis of the Dose Calculation Accuracy for IMRT in Lung: A 2D Approach," Acta Oncologica, Vol. 46, No. 7, 2007, pp. 928936. doi:10.1080/02841860701253052 